\title{
Identification and Biotechnological Application of Novel Regulatory Genes Involved in Streptomyces Polyketide Overproduction through Reverse Engineering Strategy
}

\author{
Ji-Hye Nah, Hye-Jin Kim, Han-Na Lee, Mi-Jin Lee, Si-Sun Choi, and Eung-Soo Kim \\ Department of Biological Engineering, Inha University, Incheon 402-751, Republic of Korea \\ Correspondence should be addressed to Eung-Soo Kim; eungsoo@inha.ac.kr
}

Received 13 November 2012; Revised 15 December 2012; Accepted 5 January 2013

Academic Editor: Sofiane Ghorbel

Copyright (C) 2013 Ji-Hye Nah et al. This is an open access article distributed under the Creative Commons Attribution License, which permits unrestricted use, distribution, and reproduction in any medium, provided the original work is properly cited.

Polyketide belongs to a family of abundant natural products typically produced by the filamentous soil bacteria Streptomyces. Similar to the biosynthesis of most secondary metabolites produced in the Streptomyces species, polyketide compounds are synthesized through tight regulatory networks in the cell, and thus extremely low levels of polyketides are typically observed in wild-type strains. Although many Streptomyces polyketides and their derivatives have potential to be used as clinically important pharmaceutical drugs, traditional strain improvement strategies such as random recursive mutagenesis have long been practiced with little understanding of the molecular basis underlying enhanced polyketide production. Recently, identifying, understanding, and applying a novel polyketide regulatory system identified from various Omics approaches, has become an important tool for rational Streptomyces strain improvement. In this paper, DNA microarray-driven reverse engineering efforts for improving titers of polyketides are briefly summarized, primarily focusing on our recent results of identification and application of novel global regulatory genes such as wblA, SCO1712, and SCO5426 in Streptomyces species. Sequential targeted gene manipulation involved in polyketide biosynthetic reguation synergistically provided an efficient and rational strategy for Streptomyces strain improvement. Moreover, the engineered regulation-optimized Streptomyces mutant strain was further used as a surrogate host for heterologous expression of polyketide pathway.

\section{Introduction: Streptomyces \\ Polyketide Biosynthesis and Pathway-Specific Regulation}

The high G+C Gram-positive filamentous soil bacteria Streptomyces are well known for their superior characteristics in producing a variety of secondary metabolites, including many pharmaceutically valuable compounds such as antibiotics, anticancer agents, and immunosuppressants [1, 2]. These secondary metabolites are commonly synthesized by biosynthetic enzymes, whose corresponding genes are typically clustered in the Streptomyces chromosome and are proposed to be under tight, complicated regulation at the transcriptional level [3]. Among Streptomyces secondary metabolites are polyketides, which belong to one of the largest natural product families [4]. Streptomyces polyketide biosynthesis is initiated by the key enzyme(s) referred to as polyketide synthase (PKS), which is typically classified by 3 different types. Type I PKSs are multifunctional enzymes whose domains are arranged into several modules, each of which controls incorporation of a specific precursor unit into a growing polyketide backbone during chain elongation, generating macrolide compounds such as erythromycin, tylosin, avermectin, amphotericin, and tautomycetin [5]. Type II PKSs are multienzyme complexes that perform a single set of enzymatic activities for iterative biosynthesis of aromatic products including actinorhodin, tetracycline, and doxorubicin [6]. Type III PKSs are homodimeric enzymes that catalyze iterative condensation reactions, typically known as chalcone synthase-like PKSs [7]. The genes, enzymes, and the mechanism of Streptomyces polyketide biosynthesis have been thoroughly reviewed previously [8]. In general, a simple carboxylic acid starter unit such as acetyl-CoA or propionyl-CoA is transferred to the cysteine active site of 
a $\beta$-ketoacyl synthase (KS) in the PKS complex. A specific extender unit determined by an acyl transferase (AT) domain in the PKS complex, such as a malonyl-CoA, methylmalonylCoA, or ethylmalonyl-CoA, is transferred to the thiol group of the phosphopantetheine arm of an adjacent acyl carrier protein (ACP). These subunits are joined by a decarboxylative condensation catalyzed by KS and remain covalently attached to ACP, followed by no, partial, or full series of keto group processing reactions involving $\beta$-ketoacyl reductase (KR), dehydratase $(\mathrm{DH})$, and enoyl reductase (ER). The growing chain is transferred to a downstream module of type I or iteratively recycled in type II and III for further rounds of elongation and processing to produce a full-length polyketide chain. The completed chain is typically released from the PKS by a terminal thioesterase (TE) to form a macrocyclic lactone ring or an aromatic compound.

Although a general molecular-level mechanism for polyketide biosynthesis has been relatively well characterized, a comprehensive understanding of the complex polyketide regulatory networks in Streptomyces species is yet to be elucidated [3]. The biosynthesis of Streptomyces polyketide is regulated via multiple regulatory pathways induced by both nutritional and environmental stimuli $[17,18]$. While various global regulatory systems present in most Streptomyces species are known to control both morphological differentiation and polyketide production, polyketide biosynthetic gene sets are subject to pathway-specific regulation by linked regulatory genes [19-21]. Some pathway-associated regulatory genes encode specialized types of regulatory protein, such as "SARPs" (Streptomyces antibiotic regulatory proteins) often associated with genes for type II aromatic polyketide biosynthesis $[22,23]$ and "LALs" (large ATP-binding regulators of the LuxR family) associated with some gene sets for type I macrolide polyketide biosynthesis [16]. Most of these pathway-specific regulatory genes are transcriptionally regulated by various global regulatory networks in most Streptomyces species, but their detailed mechanisms remain largely unknown.

\section{Reverse Engineering Approaches Applied in Streptomyces Strain Improvement}

Since all genome sequences of the first model species, Streptomyces coelicolor, became publically available around the year 2002, various Omics-guided strategies have been applied to increase the understanding of Streptomyces global regulatory networks involved in polyketide biosynthesis [24, 25]. Although most pathway-specific regulatory genes have been identified on the basis of their typical location within the biosynthetic pathway gene cluster, global regulatory genes are much more difficult to identify even in the well-characterized $S$. coelicolor because of the presence of more than 300 annotated putative regulatory open reading frames (ORFs) in the genome sequences $[24,25]$. Recently, Omics-guided reverse engineering approaches have emerged as an effective tool for investigating gene expression alterations associated with polyketide overproduction in several Streptomyces industrial strains [26]. This strategy enabled us to compare the recursively and randomly mutagenized overproducing industrial mutant (OIM) with the wild-type (WT) strain at the molecular gene level, eventually leading us to identify previously unknown primary and/or secondary metabolic genes critical for secondary metabolite overproduction. The S. coelicolor DNA microarray system was successfully applied to the erythromycin-overproducing Saccharopolyspora erythraea OIM as well as tylosin-overproducing $S$. fradiae OIM strains [26]. A similar genome-wide transcriptome analysis also showed that a pleiotropic antibiotic regulator, afs $S$ in S. coelicolor A3(2), and various AfsS-dependent genes are regulated by various nutritional stress responses [27]. S. peucetius DNA microarray analysis also revealed that expression patterns of $S$. peucetius genes involved in doxorubicin production change under different culture conditions [28]. In addition, genomics-driven approaches were applied to stimulate cryptic pathways such as 51-membered glycosylated macrolides, stambomycins $[29,30]$. Chemical perturbation of secondary metabolism also demonstrated important links to primary metabolism, implying that small molecules could also enhance yields of secondary metabolites for discovery and biochemical characterization [31].

\section{Identification and Manipulation of a Novel Regulatory Gene wblA in S. coelicolor}

A putative negative regulatory gene involved in polyketide biosynthesis, SCO3579, was originally proposed as a whiBlike putative transcription factor gene referred to as $w b l A$ in $S$. coelicolor [33]. whiB is one of five key regulatory genes including whiA, whiB, whiG, whiH, and whiI and known to be required for early stages of the transition of aerial hyphae to spores. Moreover, the whiB mutant exhibits poorly septated aerial hyphae with long and tightly coiled phenotype, and its expression is proposed to be repressed by a transcription factor BldD. [33]. Although whiB is a developmental regulatory gene identified and characterized in $S$. coelicolor as being essential for the sporulation of aerial hyphae, the biological function of $w b l A$ with regard to secondary metabolite regulation has not yet been examined. During the search for previously unknown polyketide regulatory genes, $w b l A$ was identified as a novel antibiotic downregulatory gene in a Streptomyces reverse-engineering approach [9]. The recursively mutated doxorubicin-overproducing $S$. peucetius OIM and the wild type $S$. peucetius subsp. caesius ATCC 27952 were examined for potential transcriptional differences between the 2 strains. After systematic analyses of growth phase-dependent transcription profiles, 20 genes with particularly large transcriptional changes between the 2 strains were selected and individually overexpressed in $S$. coelicolor under the control of the strong promoter of the Streptomyces expression vector. Among these genes, overexpression of $w b l A$ inhibited actinorhodin biosynthesis in $S$. coelicolor, and the transcript encoded by an actinorhodinspecific activator gene was reduced in $w b l A$-overexpressing $S$. coelicolor [9]. These results suggest that $w b l A$ is a broadly functioning downregulatory gene for polyketide biosynthesis in Streptomyces species. It was suggested that $\mathrm{WblA}$, which 


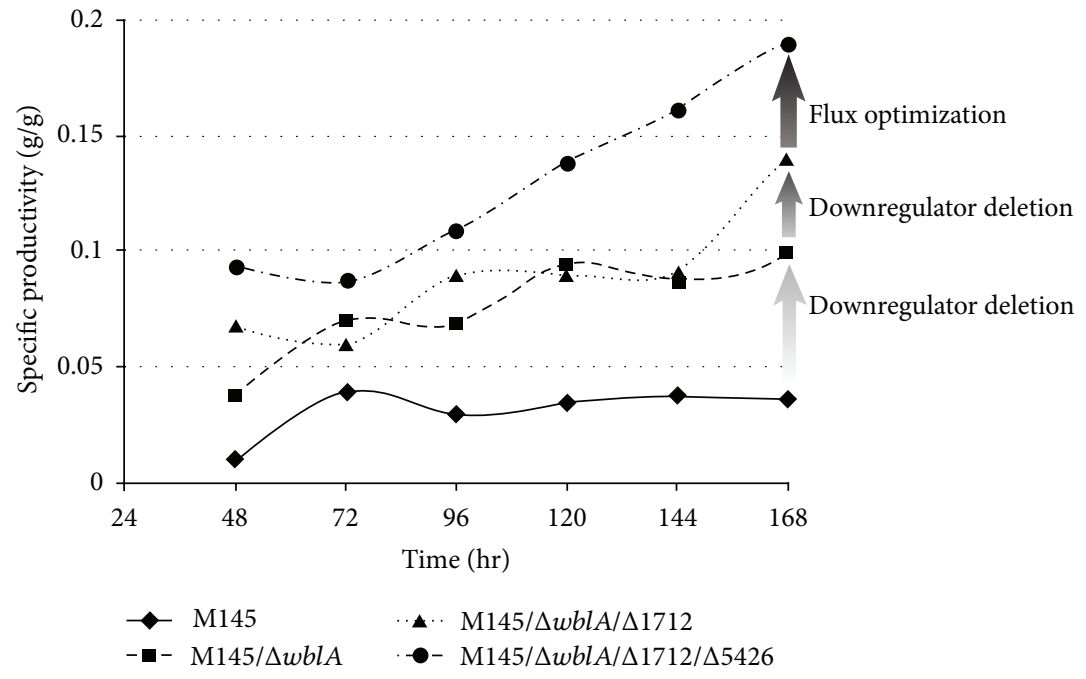

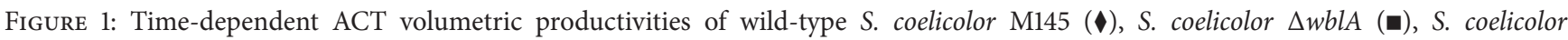
$\Delta w b l A \Delta \operatorname{SCO} 1712(\boldsymbol{\Delta})$, and S. coelicolor $\Delta$ wblA $\Delta$ SCO1712 $\Delta 5426(\bullet)$ cultured in modified R5 media during 9 days in a 2-liter bioreactor [16].

contains 4 conserved cysteine residues, may be sensitive to redox changes, perhaps via disulfide bond formation as has been found for the E. coli OxyR transcription factor $[32,34]$. Recently, transcriptome analysis with $S$. coelicolor microarray approach in a $w b l A$ mutant exhibited that approximately 180 genes involved in primary metabolism and actinorhodin biosynthesis and 100 genes related to the aerial hyphal growth were overexpressed and underexpressed, respectively [9]. WblA was then proposed to be important in the slow-down of biomass accumulation, the change from aerial hyphal initial cells to the subapical stem, apical compartments that precede sporulation, and oxidative stress response similar to that of Corynebacterium glutamicum WhcA [35]. WhcA in C. glutamicum was proved to physically bind to a specific protein named SpiA (stress protein interacting with WhcA) only under the nonoxidative condition. Interestingly, a SpiA ortholog present in S. coelicolor also showed a similar WblASpiA interaction in the oxidative stress response in $S$. coelicolor, and a global regulatory protein called AdpA (A-factordependent protein A) might control the wblA expression through direct binding to the promoter region of $w b l A$ (Lee et al., unpublished data). Currently, several $w b l A$ ortholog genes have been identified in various Streptomyces species, and they are presumably involved in secondary metabolite regulation (Table 1).

In addition to identifying $w b l A$, comparative microarray analysis revealed that SCO1712 expression was considerably lower in actinorhodin overproducing S. coelicolor M145 than in the actinorhodin less-producing S. coelicolor J1501 [36]. SCO1712 encodes a 205-amino acid protein with an $\mathrm{N}$ terminal TetR-family helix-turn-helix (HTH) DNA-binding domain, whose biological function related to secondary metabolite regulation has not been reported. A significant decrease in the blue pigment actinorhodin was observed in the SCO1712-expressing S. coelicolor in plate culture.
Moreover, the transcription level of actinorhodin pathwayspecific actII-ORF4 was significantly increased from the S. coelicolor M145 $\triangle \mathrm{SCO} 1712$, while an opposite transcription pattern was observed in the SCO1712-overexpressing S. coelicolor M145 strain, implying that SCO1712 had a global inhibitory effect on polyketide biosynthesis in $S$. coelicolor [36]. Interestingly, the expression of not only $w b l A$ but also SCO1712 complemented the $\Delta w b l A$ mutant phenotype independently. These results suggest that $w b l A$ may not be required for SCO1712 to downregulate polyketide biosynthesis. SCO1712 was additionally disrupted in an S. coelicolor M145 $\Delta$ wblA mutant strain; the S. coelicolor M145 $\Delta$ wblA $\Delta$ SCO1712 double knock-out mutant strain; exhibited considerably higher actinorhodin volumetric productivity (Figure 1). This implies that SCO1712 is not directly related to $w b l A$ function and more likely encodes a $w b l A$ independent polyketide downregulator [36]. These results suggest that sequential targeted gene disruptions of independently working downregulatory genes may be an efficient and rational strategy for Streptomyces strain improvement.

\section{Identification and Engineering of $w b l A$ Ortholog in S. peucetius Industrial Mutant}

To examine whether the $w b l A$ ortholog is also present in other Streptomyces strains with similar biological significance in polyketide regulation, a total genomic DNA library from the doxorubicin-producing $S$. peucetius OIM was constructed. Cosmid library construction and screening successfully generated one positive candidate containing the entire $w b l A$ ortholog gene $\left(w b l A_{\text {spe }}\right)$ [10]. Sequence analysis of $345 \mathrm{bp}$ ORF containing $w b l A_{\text {spe }}$ showed a protein-coding sequence showing a high degree of amino acid similarity with the translated products of $w b l A$ genes from several previously reported 
TABLE 1: wblA orthologs identified from various Streptomyces species.

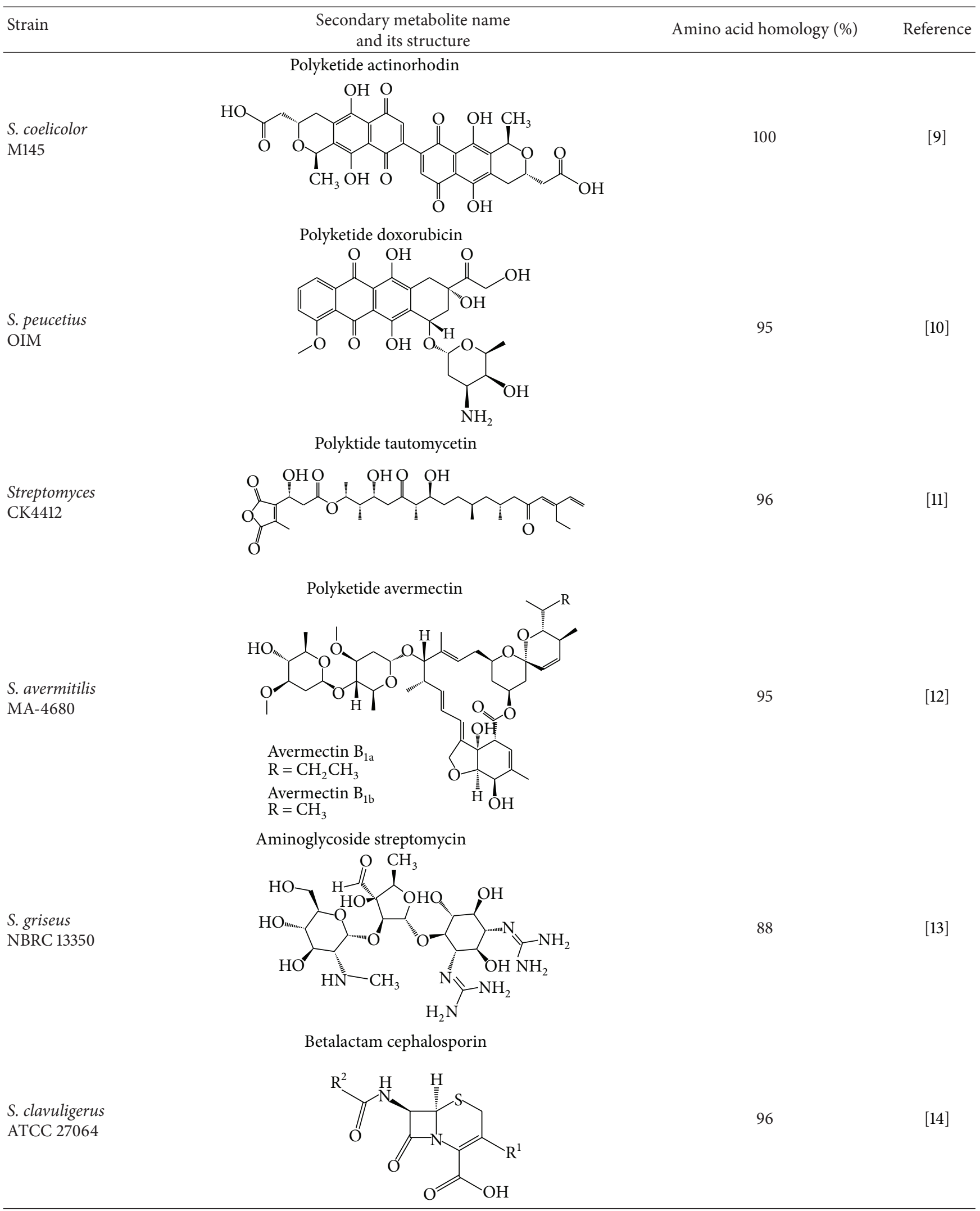


TABle 1: Continued.

Strain Amino acid homology (\%)

Streptomyces genomes, including S. coelicolor (95\%), S. avermitilis MA-4680 (92\%), S. griseus NBRC 13350 (96\%), and S. clavuligerus ATCC 27064 (91\%). As expected, approximately $35 \%$ more doxorubicin and $150 \%$ more 14 -deoxydoxorubicin (daunorubicin) were produced by $S$. peucetius OIM $\Delta w b l A_{\text {spe }}$ compared with the $S$. peucetius OIM [10].

Through a second round of microarray analysis between S. peucetius OIM and OIM $\Delta w b l A_{\text {spe }}$, six genes showing more than 4-fold transcriptional changes between these two strains were identified, followed by expression in $S$. coelicolor [10]. Since the only S. coelicolor exconjugant containing the SCO4967 construct produced approximately 2fold more actinorhodin than the control, the Streptomyces expression vector pSET152 derivative containing SCO4967 was reintroduced into $S$. peucetius OIM $\Delta w b l A_{\text {spe }}$. SCO 4967 is listed to encode a conserved hypothetical protein with little information of in vivo biological function. SCO4967 overexpression in $S$. peucetius $\mathrm{OIM} \Delta w b l A_{\text {spe }}$ resulted in approximately 1.7-fold more aklavinone (another doxorubicin precursor polyketide) productivity than that of $S$. peucetius OIM $\Delta w b l A_{\text {spe }}$ exconjugant harboring an empty vector [10]. Moreover, the SCO4967-containing S. peucetius $\mathrm{OIM} \Delta w b l A_{\text {spe }}$ strain exhibited the highest total volumetric production of doxorubicin/daunorubicin/aklavinone, which was approximately 1.9-fold and 1.3-fold higher than those of $S$. peucetius OIM and S. peucetius OIM $\Delta w b l A_{\text {spe }}$, respectively (Figure 2). This implies that sequential genetic manipulation of target genes identified through interspecies comparative microarray analysis may be an efficient and rational strategy for Streptomyces strain improvement [10, 37].

\section{Identification and Engineering of $w b l A$ Ortholog in Streptomyces sp. CK4412}

To isolate another $w b l A$ ortholog gene from a type I polyketide-producing Streptomyces species, a total genomic DNA library from Streptomyces sp. CK4412 was screened using PCR degenerate primers based on the highly conserved regions present in both the $w b l A$ sequences of $S$. coelicolor A3(2) and S. avermitilis ATCC31780 [11, 38]. Streptomyces sp. CK4412 has been known to produce an unusual linear polyketide compound named tautomycetin (TMC), which inhibits $\mathrm{T}$ cell proliferation at concentrations 100 -fold lower than those needed to achieve maximal inhibition with cyclosporin A. Since TMC is believed to specifically block tyrosine phosphorylation of intracellular signal mediators downstream of Src tyrosine kinases in a T cell-specific manner, TMC is a novel potent T cell-specific immunosuppressive agent whose mechanism of action is different from that of cyclosporin A or FK506. [39-41]. Through Streptomyces sp. CK4412 DNA cosmid library screening, one positive cosmid containing the entire $w b l A$ ortholog gene (named $w b l A_{t m c}$ ) was selected. Complete sequence analysis revealed that the 390-bp $w b l A_{t m c}$ gene encodes a 130-amino acid protein with a high degree of amino acid similarity with that of the translated wblA gene products from S. coelicolor (96\%), S. avermitilis MA-4680 (93\%), S. griseus NBRC 13350 (89\%), and S. clavuligerus ATCC 27064 (93\%), all of which contain 4 conserved cysteine residues and a helix-turn-helix (HTH) motif [11].

Although in silico sequence analyses of $w b l A_{t m c}$ from Streptomyces sp. CK4412 was consistent with its putative regulatory roles in other Streptomyces polyketide biosyntheses, the in vivo function of $w b l A_{t m c}$ was confirmed using a gene disruption approach. Construction of the wblA mutant (Streptomyces sp. CK4412-001) was generated using PCRtargeted disruption followed by PCR analysis confirmation. Culture broths of Streptomyces spp. CK4412, CK4412-001, CK4412-001/wblA $A_{t m c}$, and CK4412/wblA tmc grown in MS media were extracted, these were analyzed using an antifungal bioassay, and the presence of TMC was quantified using high-pressure liquid chromatography (HPLC). Streptomyces CK4412-001 produced approximately 3-fold more TMC compared to WT Streptomyces CK4412 (Figure 3). Significantly enhanced antifungal activity against Aspergillus niger was also observed in the extracts of Streptomyces sp. CK4412-001 under the same culture conditions. Moreover, an 


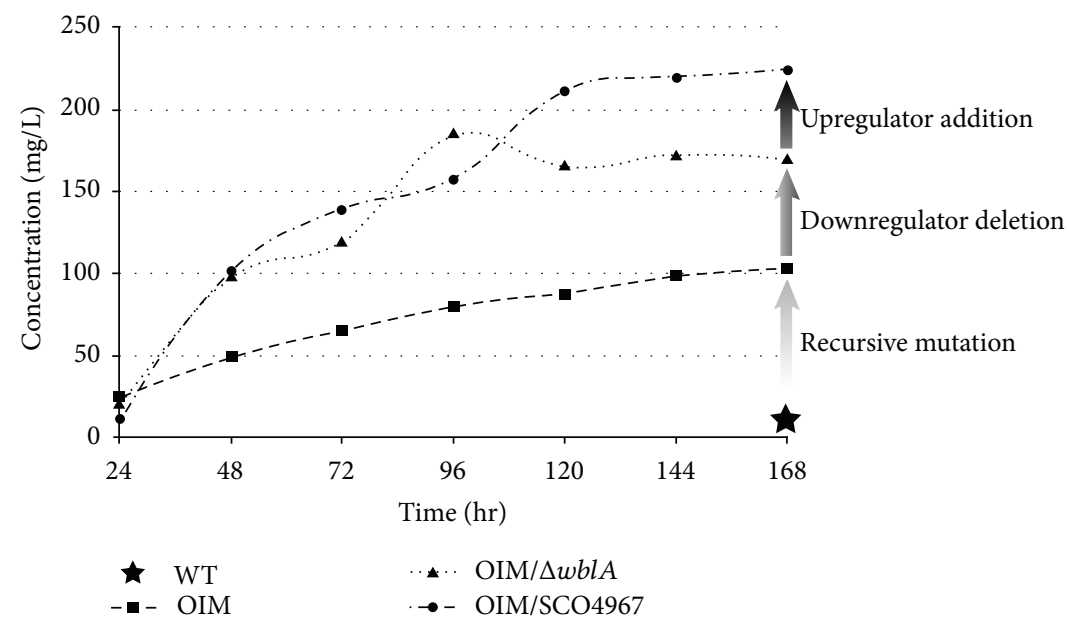

FIgURE 2: Time-dependent DXR/DNR/aklavinone volumetric productivities of S. peucetius WT ( $\star$ ), S. peucetius OIM (匹), S. peucetius $\mathrm{OIM} \Delta w b l A_{\text {spe }}(\mathbf{\Delta})$, and S. peucetius OIM $\Delta w b l A_{\text {spe }} / \mathrm{SCO} 4967(\bullet)[32]$.

integrating conjugative vector, into which the coding region of $w b l A_{t m c}$ and its own upstream promoter region were cloned, was constructed (pSETHYGwblA). Both HPLC and a bioassay confirmed that TMC productivity and antifungal activity were reduced in the Streptomyces sp. CK4412-001 mutant strain carrying pSETHYGwblA back to the WT level, implying that $w b l A_{t m c}$ also plays a global antibiotic downregulatory role in type I polyketide biosynthesis in Streptomyces sp. CK4412 (Figure 3).

\section{Synergistic Redesign of Polyketide and Precursor Flux Regulatory Pathways}

Comparative transcriptome analysis between $S$. coelicolor WT and an S. coelicolor $\Delta w b l A \Delta$ SCO1712 double knock-out mutant revealed an additional 14 genes that displayed no particular (less than 1.2-fold) transcriptional changes [42]. These putative wblA/SCO1712-independent genes include a carbon flux-regulating SCO5426, which is one of the 3 6-phosphofructokinase genes. SCO5426 disruption was previously reported to enhance both precursor carbon flux and NADPH supply for polyketide biosynthesis by activating the pentose phosphate pathway, resulting in significantly enhanced actinorhodin production in S. coelicolor [43]. Based on the above observations, additional deletion of SCO5426 in the S. coelicolor $\Delta w b l A \Delta$ SCO1712 double knockout mutant may further enhance actinorhodin precursor flux as well as NADPH supply in S. coelicolor. While all mutant strains exhibited comparable growth patterns, the $S$. coelicolor $\Delta w b l A \Delta$ SCO1712 $\Delta$ SCO5426 triple knock-out mutant strain exhibited the highest actinorhodin productivity, which was 1.7-fold and 1.3-fold higher than those of the single knockout $S$. coelicolor $\triangle w b l A$ and the double knock-out $S$. coelicolor $\Delta w b l A \Delta \mathrm{SCO} 1712$ mutant strains, respectively [42]. These results suggest that sequential targeted gene disruption of independently working downregulators as well as precursor flux downregulators involved in polyketide biosynthesis may synergistically provide an efficient and rational strategy for Streptomyces strain improvement.

\section{Potential Application as a Surrogate Host for Synthetic Biology}

As a complementary strategy for valuable Streptomyces polyketide production, functional expression of the target polyketide pathway in a Streptomyces heterologous host has been applied. Several secondary metabolite pathways were expressed in relatively well-characterized and genetically amenable Streptomyces surrogate hosts including S. albus, S. lividans, S. coelicolor, S. avermitilis, S. ambofaciens, $S$. roseosporus, and S. grisefuscus $[44,45]$. Recently, the genomes of these strains have been further engineered to maximize foreign polyketide production by deleting some endogenous biosynthetic gene clusters and/or preventing diversion of precursors into competing secondary metabolic pathways. Especially, a genome-minimized $S$. avermitilis industrial mutant strain was also used successfully for foreign polyketide pathway expression as a heterologous expression host [46], suggesting a realistic alternative strategy for overproducing exogenous natural and unnatural polyketides.

A polyketide nonproducing $S$. coelicolor mutant strain was generated by deleting the entire actinorhodin cluster from the chromosome of a previously generated $S$. coelicolor $\Delta w b l A \Delta$ SCO1712 $\Delta$ SCO5426 triple knock-out mutant strain, which was shown to stimulate actinorhodin biosynthesis through deletion of 2 antibiotic down-regulators as well as a polyketide precursor flux downregulator [47]. Using this engineered $S$. coelicolor mutant strain as a surrogate host or a cell factory from a synthetic biology perspective, a model minimal polyketide pathway for aloesaponarin II [48] was cloned and functionally expressed in a highcopy expression plasmid, followed by quantitative polyketide analysis. As expected, aloesaponarin II production was 

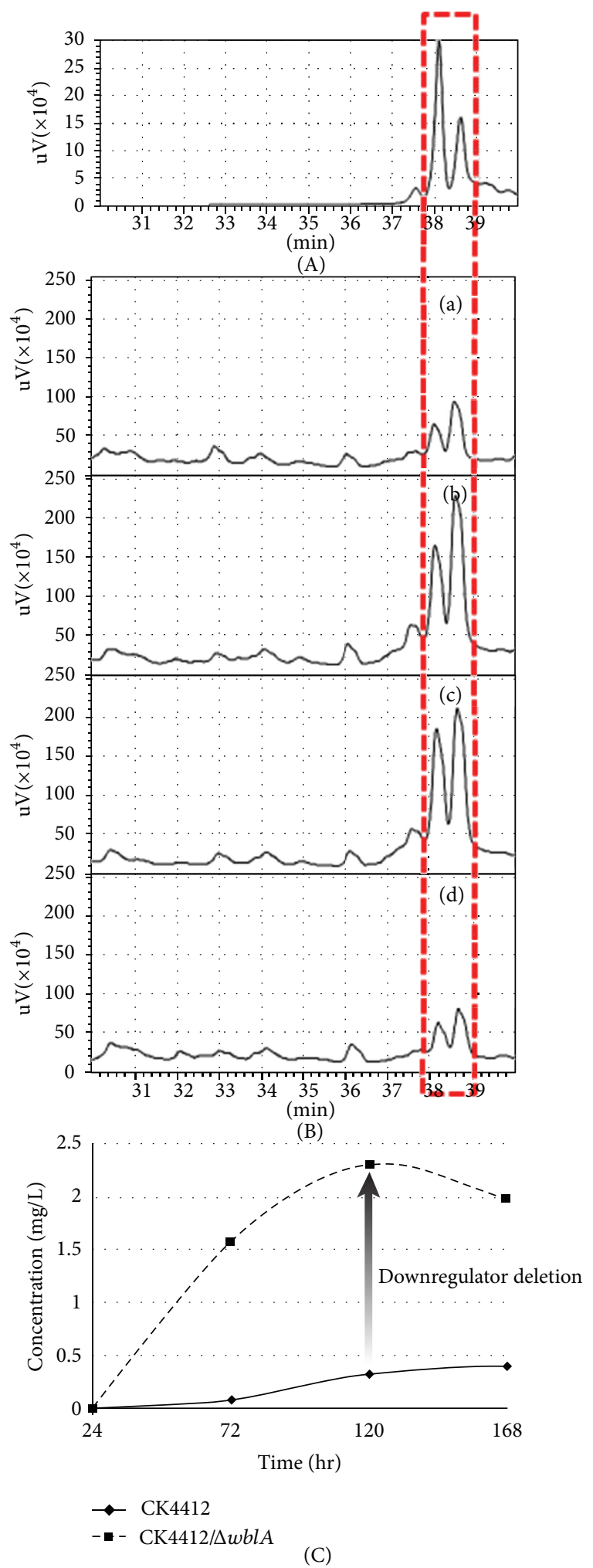

Figure 3: (A) Authentic TMC standard. (B) TMC volumetric productivities measured by quantitative HPLC analyses of the ethyl acetateextracted culture broths were $1.69 \mathrm{mg} \cdot \mathrm{L}^{-1}$ for the wild-type strain CK4412 (a), $5.44 \mathrm{mg} \cdot \mathrm{L}^{-1}$ for the wblA-tmc disruptant (b), $4.04 \mathrm{mg} \cdot \mathrm{L}^{-1}$ for CK4412-001/wblA-tmc (c), and $1.41 \mathrm{mg} \cdot \mathrm{L}^{-1}$ for CK4412/wblA-tmc (d). (C) Time-dependent tautomycetin volumetric productivities of

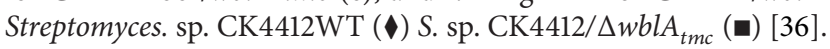




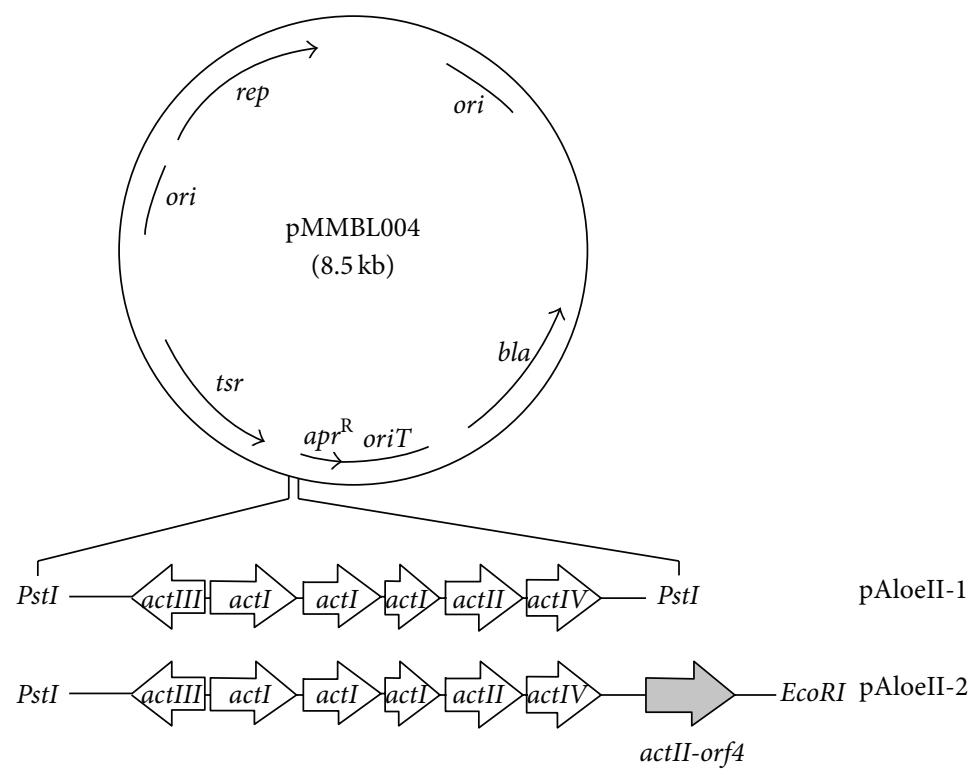

(a)

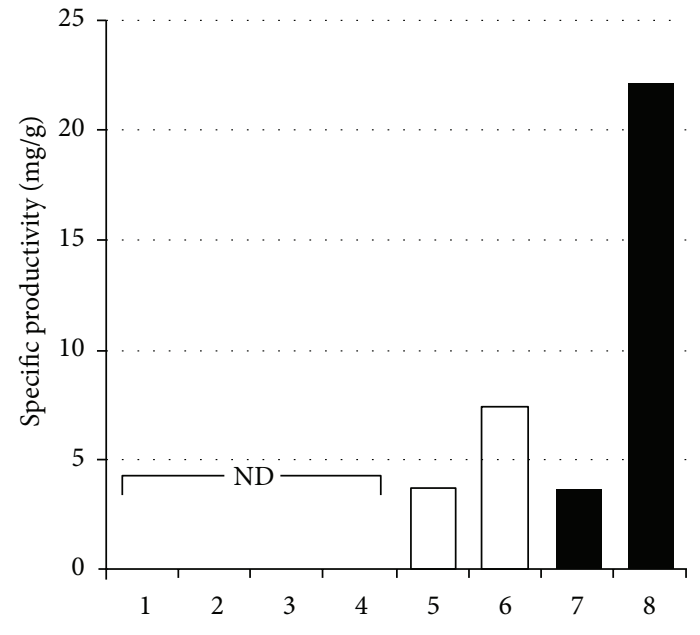

(c)

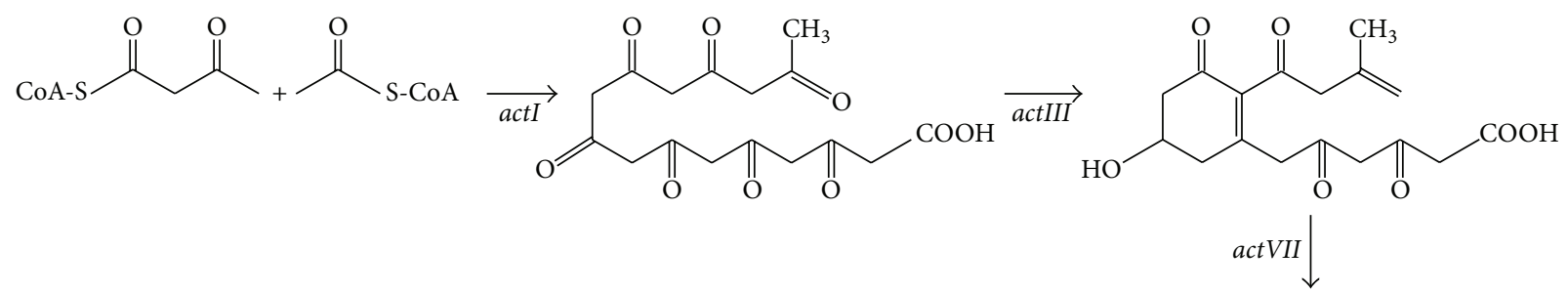<smiles>[R]c1c(O)cc2c(c1C)C(=O)c1c(O)cccc1C2=O</smiles><smiles>CC(=O)C1C(=O)c2c(O)cccc2CC1(O)CC(O)CC(=O)O</smiles>

(b)

Figure 4: (a) Plasmid map of the pMMBL004, pAloeII-1 (pMMBL004 containing PstI fragment), and pAloeII-2 (pAloeII-1 containing actIIorf4); rep: replicon; ori: origin of replication; bla: $\beta$-lactamase ${ }^{\mathrm{R}}$; tsr: apramycin ${ }^{\mathrm{R}}$; apr: apramycin ${ }^{\mathrm{R}}$; oriT: origin of transfer. (b) Schematic representation of the aloesaponarin II biosynthetic pathway. Solid and dotted arrows represent presumed enzymatic and spontaneous steps, respectively. 3,8-DMAC: 3,8-dihydroxy-1-methyl-anthraquinone-2-carboxylic acid. R1 groups of aloesaponarin II and 3,8-DMAC are hydrogen and carboxylic acid, respectively. (c) Productions of aloesaponarin II and 3,8-DMAC in recombinant $S$. coelicolor strains. 1, $S$. coelicolor $\triangle \mathrm{ACT} / \mathrm{pMMBL} 004 ; 2$, S. coelicolor $\triangle \mathrm{ACT} \Delta$ wblA $\Delta$ SCO1712 $\Delta$ SCO5426/pMMBL004; 3, S. coelicolor $\Delta$ ACT/pAloeII-1; 4, S. coelicolor $\Delta$ ACT $\Delta$ wblA $\Delta$ SCO1712 $\Delta$ SCO5426/pAloeII-1; 5, S. coelicolor $\Delta$ ACT/pAloeII-2; 6, S. coelicolor $\Delta$ ACT $\Delta$ wblA $\Delta$ SCO1712 $\Delta$ SCO5426/pAloeII-2; 7, S. coelicolor $\Delta$ ACT/pAloeII-2; 8, S. coelicolor $\Delta$ ACT $\Delta$ wblA $\Delta$ SCO1712 $\Delta$ SCO5426/pAloeII-2; 1 6, liquid culture; 7 8, solid culture; ND, not detected [29].

observed at the highest level in the actinorhodin clusterdeleted and downregulators-deleted mutant strain, S. coelicolor $\triangle \mathrm{ACT} \Delta$ wblA $\triangle \mathrm{SCO} 1712 \Delta \mathrm{SCO} 5426$ (Figure 4). These results imply that this engineered actinorhodin-free and regulation-optimized $S$. coelicolor mutant strain can be used as a general surrogate for efficiently expressing foreign polyketide pathways. In conclusion, biotechnological applications of the independently functioning regulatory pathway identified through microarray-driven reverse engineering strategy may be beneficial for Streptomyces strain improvement for polyketide overproduction as well as for efficient host cell factory construction for synthetic biology.

\section{Acknowledgment}

This work was supported by Grant no. D00040 from the Global Research Network (GRN) Program of the National Research Foundation of Korea.

\section{References}

[1] R. H. Baltz, "Molecular engineering approaches to peptide, polyketide and other antibiotics," Nature Biotechnology, vol. 24, no. 12, pp. 1533-1540, 2006.

[2] D. A. Hopwood, "Soil to genomics: the Streptomyces chromosome," Annual Review of Genetics, vol. 40, pp. 1-23, 2006. 
[3] M. J. Bibb, "Regulation of secondary metabolism in streptomycetes," Current Opinion in Microbiology, vol. 8, no. 2, pp. 208215, 2005.

[4] C. Khosla, "Structures and mechanisms of polyketide synthases," Journal of Organic Chemistry, vol. 74, no. 17, pp. 64166420, 2009.

[5] L. Katz and S. Donadio, "Polyketide synthesis: prospects for hybrid antibiotics," Annual Review of Microbiology, vol. 47, pp. 875-912, 1993.

[6] C. Hertweck, A. Luzhetskyy, Y. Rebets, and A. Bechthold, “Type II polyketide synthases: gaining a deeper insight into enzymatic teamwork," Natural Product Reports, vol. 24, no. 1, pp. 162-190, 2007.

[7] K. Watanabe, A. P. Praseuth, and C. C. Wang, "A comprehensive and engaging overview of the type III family of polyketide synthases," Current Opinion in Chemical Biology, vol. 11, no. 3, pp. 279-286, 2007.

[8] H. G. Floss, "Combinatorial biosynthesis-Potential and problems," Journal of Biotechnology, vol. 124, no. 1, pp. 242-257, 2006.

[9] S. H. Kang, J. Huang, H. N. Lee, Y. A. Hur, S. N. Cohen, and E. S. Kim, "Interspecies DNA microarray analysis identifies wblA as a pleiotropic down-regulator of antibiotic biosynthesis in Streptomyces," Journal of Bacteriology, vol. 189, no. 11, pp. 43154319, 2007.

[10] J. H. Noh, S. H. Kim, H. N. Lee, S. Y. Lee, and E. S. Kim, "Isolation and genetic manipulation of the antibiotic downregulatory gene, $w b l A$ ortholog for doxorubicin-producing Streptomyces strain improvement," Applied Microbiology and Biotechnology, vol. 86, no. 4, pp. 1145-1153, 2010.

[11] J. H. Nah, S. H. Park, H. M. Yoon, S. S. Choi, C. H. Lee, and E. S. Kim, "Identification and characterization of $w b l A-$ dependent tmcT regulation during tautomycetin biosynthesis in Streptomyces sp. CK4412," Biotechnology Advances, vol. 30, no. 1, pp. 202-209, 2012.

[12] H. Ikeda, J. Ishikawa, A. Hanamoto et al., "Complete genome sequence and comparative analysis of the industrial microorganism Streptomyces avermitilis," Nature Biotechnology, vol. 21, no. 5, pp. 526-531, 2003.

[13] Y. Ohnishi, J. Ishikawa, H. Hara et al., "Genome sequence of the streptomycin-producing microorganism Streptomyces griseus IFO 13350," Journal of Bacteriology, vol. 190, no. 11, pp. 40504060, 2008.

[14] M. H. Medema, A. Trefzer, A. Kovalchuk, M. van den Berg, U. Muller, W. Heijne et al., "The sequence of a 1.8-mb bacterial linear plasmid reveals a rich evolutionary reservoir of secondary metabolic pathways," Genome Biology and Evolution, vol. 2, pp. 212-224, 2010.

[15] M. Rabyk, B. Ostash, Y. Rebets, S. Walker, and V. Fedorenko, "Streptomyces ghanaensis pleiotropic regulatory gene $w b l A_{g h}$ influences morphogenesis and moenomycin production," Biotechnology Letters, vol. 33, no. 12, pp. 2481-2486, 2011.

[16] D. J. Wilson, Y. Xue, K. A. Reynolds, and D. H. Sherman, "Characterization and analysis of the pikD regulatory factor in the pikromycin biosynthetic pathway of Streptomyces venezuelae," Journal of Bacteriology, vol. 183, no. 11, pp. 3468-3475, 2001.

[17] E. J. Lee, N. Karoonuthaisiri, H. S. Kim et al., "A master regulator $\sigma \mathrm{B}$ governs osmotic and oxidative response as well as differentiation via a network of sigma factors in Streptomyces coelicolor," Molecular Microbiology, vol. 57, no. 5, pp. 1252-1264, 2005.

[18] G. P. Van Wezel and K. J. McDowall, "The regulation of the secondary metabolism of Streptomyces: new links and experimental advances," Natural Product Reports, vol. 28, no. 7, pp. 1311-1333, 2011.

[19] F. Lombó, A. F. Braña, C. Méndez, and J. A. Salas, "The mithramycin gene cluster of Streptomyces argillaceus contains a positive regulatory gene and two repeated DNA sequences that are located at both ends of the cluster," Journal of Bacteriology, vol. 181, no. 2, pp. 642-647, 1999.

[20] L. Retzlaff and J. Distler, "The regulator of streptomycin gene expression, StrR, of Streptomyces griseus is a DNA binding activator protein with multiple recognition sites," Molecular Microbiology, vol. 18, no. 1, pp. 151-162, 1995.

[21] L. Tang, A. Grimm, Y. X. Zhang, and C. R. Hutchinson, "Purification and characterization of the DNA-binding protein Dnrl, a transcriptional factor of daunorubicin biosynthesis in Streptomyces peucetius," Molecular Microbiology, vol. 22, no. 5, pp. 801-813, 1996.

[22] P. Arias, M. A. Fernández-Moreno, and F. Malpartida, "Characterization of the pathway-specific positive transcriptional regulator for actinorhodin biosynthesis in Streptomyces coelicolor A3(2) as a DNA-binding protein," Journal of Bacteriology, vol. 181, no. 22, pp. 6958-6968, 1999.

[23] A. Wietzorrek and M. Bibb, "A novel family of proteins that regulates antibiotic production in Streptomycetes appears to contain an OmpR-like DNA-binding fold," Molecular Microbiology, vol. 25, no. 6, pp. 1181-1184, 1997.

[24] S. D. Bentley, K. F. Chater, A. M. Cerdeno-Tarraga, G. L. Challis, N. R. Thomson, K. D. James et al., "Complete genome sequence of the model actinomycete Streptomyces coelicolor A3(2)," Nature, vol. 417, no. 6885, pp. 141-147, 2002.

[25] J. Huang, C. J. Lih, K. H. Pan, and S. N. Cohen, "Global analysis of growth phase responsive gene expression and regulation of antibiotic biosynthetic pathways in Streptomyces coelicolor using DNA microarrays," Genes and Development, vol. 15, no. 23, pp. 3183-3192, 2001.

[26] A. M. Lum, J. Huang, C. R. Hutchinson, and C. M. Kao, "Reverse engineering of industrial pharmaceutical-producing actinomycete strains using DNA microarrays," Metabolic Engineering, vol. 6, no. 3, pp. 186-196, 2004.

[27] W. Lian, K. P. Jayapal, S. Charaniya et al., "Genome-wide transcriptome analysis reveals that a pleiotropic antibiotic regulator, AfsS, modulates nutritional stress response in Streptomyces coelicolor A3(2)," BMC Genomics, vol. 9, article 56, 2008.

[28] J. R. Yang, E. Song, B. G. Kim, E. S. Kim, J. K. Sohng, and M. K. Oh, "Expression profiling of Streptomyces peucetius metabolic genes using DNA microarray analysis," Biotechnology and Bioprocess Engineering, vol. 13, no. 6, pp. 738-744, 2008.

[29] K. Ochi and T. Hosaka, "New strategies for drug discovery: activation of silent or weakly expressed microbial gene clusters," Applied Microbiology and Biotechnology, vol. 97, no. 1, pp. 87-98, 2013.

[30] L. Laureti, L. Song, S. Huang et al., "Identification of a bioactive 51-membered macrolide complex by activation of a silent polyketide synthase in Streptomyces ambofaciens," Proceedings of the National Academy of Sciences of the United States of America, vol. 108, no. 15, pp. 6258-6263, 2011.

[31] A. Craney, C. Ozimok, S. M. Pimentel-Elardo, A. Capretta, and J. R. Nodwell, "Chemical perturbation of secondary metabolism demonstrates important links to primary metabolism," Chemistry \& Biology, vol. 19, pp. 1020-1027, 2012.

[32] P. Jakimowicz, M. R. Cheesman, W. R. Bishai, K. F. Chater, A. J. Thomson, and M. J. Buttner, "Evidence that the Streptomyces 
developmental protein WhiD, a member of the WhiB family, binds a [4Fe-4S] cluster," Journal of Biological Chemistry, vol. 280, no. 9, pp. 8309-8315, 2005.

[33] J. A. Soliveri, J. Gomez, W. R. Bishai, and K. F. Chater, "Multiple paralogous genes related to the Streptomyces coelicolor developmental regulatory gene whiB are present in Streptomyces and other Actinomycetes," Microbiology, vol. 146, no. 2, pp. 333$343,2000$.

[34] K. Fowler-Goldsworthy, B. Gust, S. Mouz, G. Chandra, K. C. Findlay, and K. F. Chater, "The actinobacteria-specific gene wblA controls major developmental transitions in Streptomyces coelicolor A3(2)," Microbiology, vol. 157, no. 5, pp. 1312-1328, 2011.

[35] J. S. Kim, H. N. Lee, P. Kim, H. S. Lee, and E. S. Kim, "Negative role of $w b l A$ in response to oxidative stress in Streptomyces coelicolor," Journal of Microbiology and Biotechnology, vol. 22, no. 6, pp. 736-741, 2012.

[36] H. N. Lee, J. Huang, J. H. Im et al., "Putative TetR family transcriptional regulator SCO1712 encodes an antibiotic downregulator in Streptomyces coelicolor," Applied and Environmental Microbiology, vol. 76, no. 9, pp. 3039-3043, 2010.

[37] N. P. Niraula, S. H. Kim, J. K. Sohng, and E. S. Kim, "Biotechnological doxorubicin production: pathway and regulation engineering of strains for enhanced production," Applied Microbiology and Biotechnology, vol. 87, no. 4, pp. 1187-1194, 2010.

[38] S. S. Choi, Y. A. Hur, D. H. Sherman, and E. S. Kim, "Isolation of the biosynthetic gene cluster for tautomycetin, a linear polyketide T cell-specific immunomodulator from Streptomyces sp. CK4412," Microbiology, vol. 153, no. 4, pp. 1095-1102, 2007.

[39] X. C. Cheng, T. Kihara, X. Ying et al., "A new antibiotic, tautomycetin," Journal of Antibiotics, vol. 42, no. 1, pp. 141-144, 1989.

[40] J. H. Shim, H. K. Lee, E. J. Chang et al., "Immunosuppressive effects of tautomycetin in vivo and in vitro via $\mathrm{T}$ cell-specific apoptosis induction," Proceedings of the National Academy of Sciences of the United States of America, vol. 99, no. 16, pp. 1061710622, 2002.

[41] J. H. Lee, J. S. Lee, S. E. Kim et al., "Tautomycetin inhibits growth of colorectal cancer cells through $\mathrm{p} 21^{\text {cip/WAF1 }}$ induction via the extracellular signal-regulated kinase pathway," Molecular Cancer Therapeutics, vol. 5, no. 12, pp. 3222-3231, 2006.

[42] S. H. Kim, H. N. Lee, H. J. Kim, and E. S. Kim, “Transcriptome analysis of an antibiotic downregulator mutant and synergistic actinorhodin stimulation via disruption of a precursor flux regulator in Streptomyces coelicolor," Applied and Environmental Microbiology, vol. 77, no. 5, pp. 1872-1877, 2011.

[43] I. Borodina, J. Siebring, J. Zhang et al., "Antibiotic overproduction in Streptomyces coelicolor A3(2) mediated by phosphofructokinase deletion," Journal of Biological Chemistry, vol. 283, no. 37, pp. 25186-25199, 2008.

[44] J. P. Gomez-Escribano and M. J. Bibb, "Engineering Streptomyces coelicolor for heterologous expression of secondary metabolite gene clusters," Microbial Biotechnology, vol. 4, no. 2, pp. 207-215, 2011.

[45] R. H. Baltz, "Streptomyces and Saccharopolyspora hosts for heterologous expression of secondary metabolite gene clusters," Journal of Industrial Microbiology and Biotechnology, vol. 37, no. 8, pp. 759-772, 2010.

[46] M. Komatsu, T. Uchiyama, S. Omura, D. E. Cane, and H. Ikeda, "Genome-minimized Streptomyces host for the heterologous expression of secondary metabolism," Proceedings of the
National Academy of Sciences of the United States of America, vol. 107, no. 6, pp. 2646-2651, 2010.

[47] H. N. Lee, H. J. Kim, P. Kim, H. S. Lee, and E. S. Kim, "Minimal polyketide pathway expression in an actinorhodin cluster-deleted and regulation-stimulated Streptomyces coelicolor," Journal of Industrial Microbiology and Biotechnology, vol. 39, no. 5, pp. 805-811, 2012.

[48] E. S. Kim, K. D. Cramer, A. L. Shreve, and D. H. Sherman, "Heterologous expression of an engineered biosynthetic pathway: functional dissection of type II polyketide synthase components in Streptomyces species," Journal of Bacteriology, vol. 177, no. 5, pp. 1202-1207, 1995. 

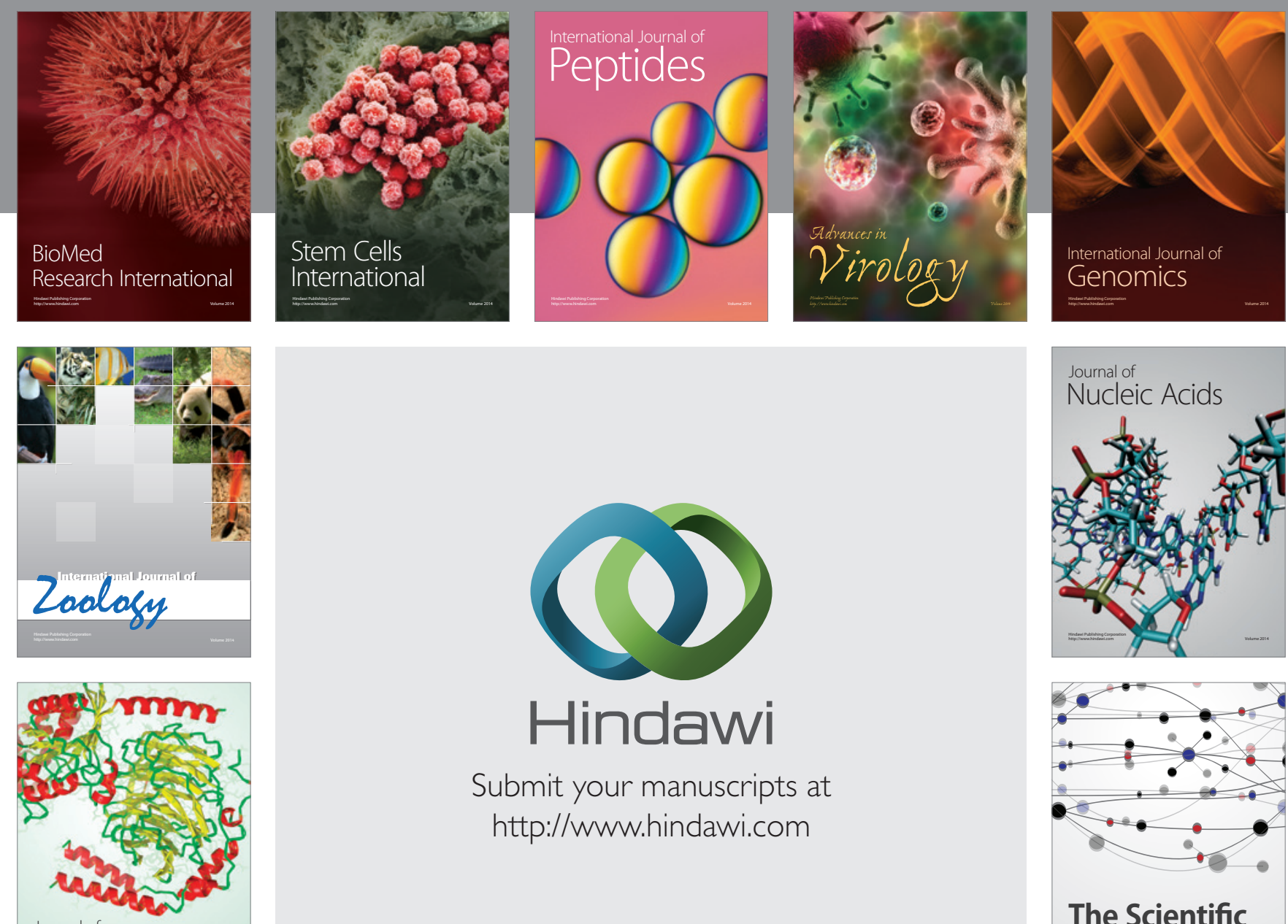

Submit your manuscripts at

http://www.hindawi.com

Journal of
Signal Transduction
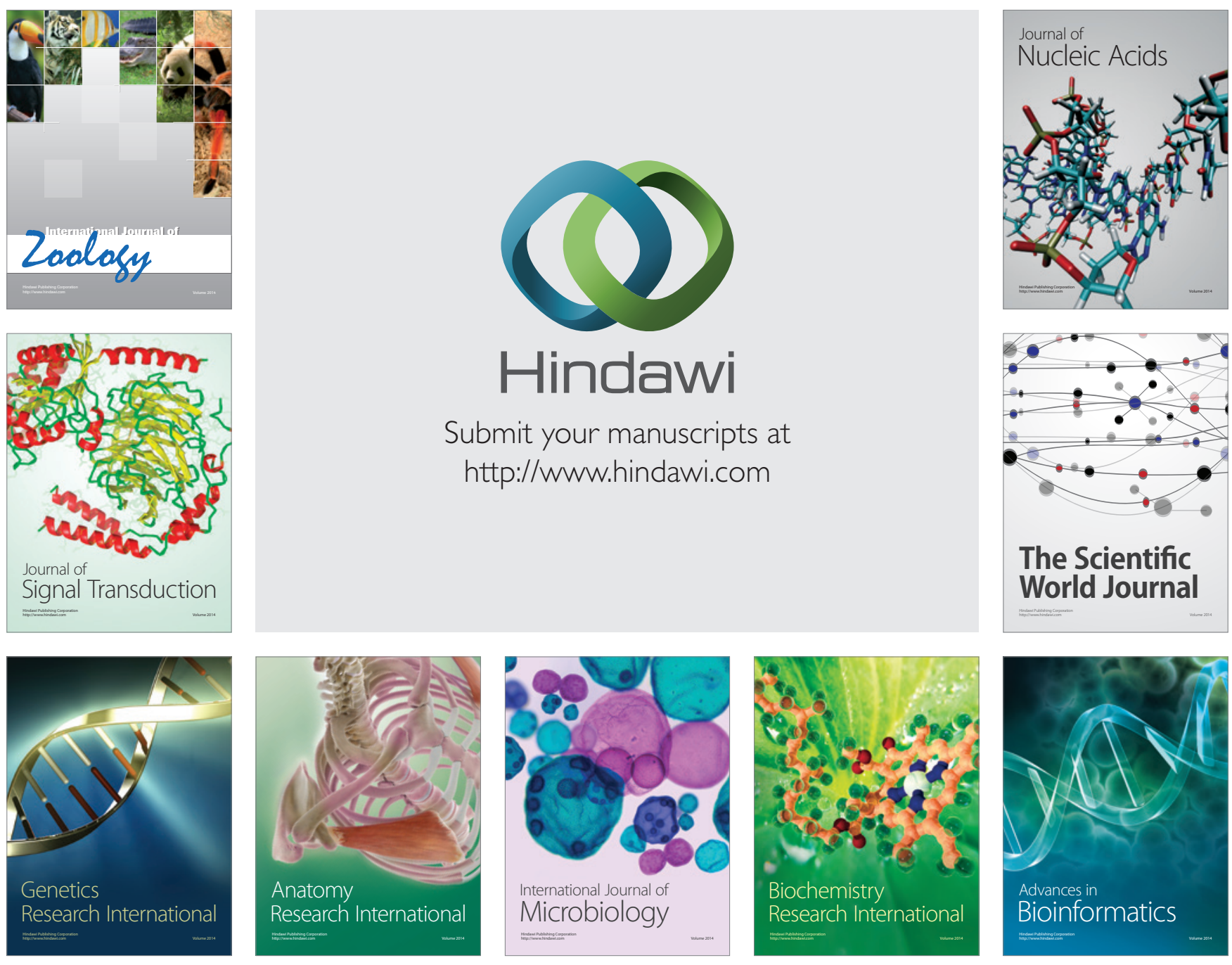

The Scientific World Journal
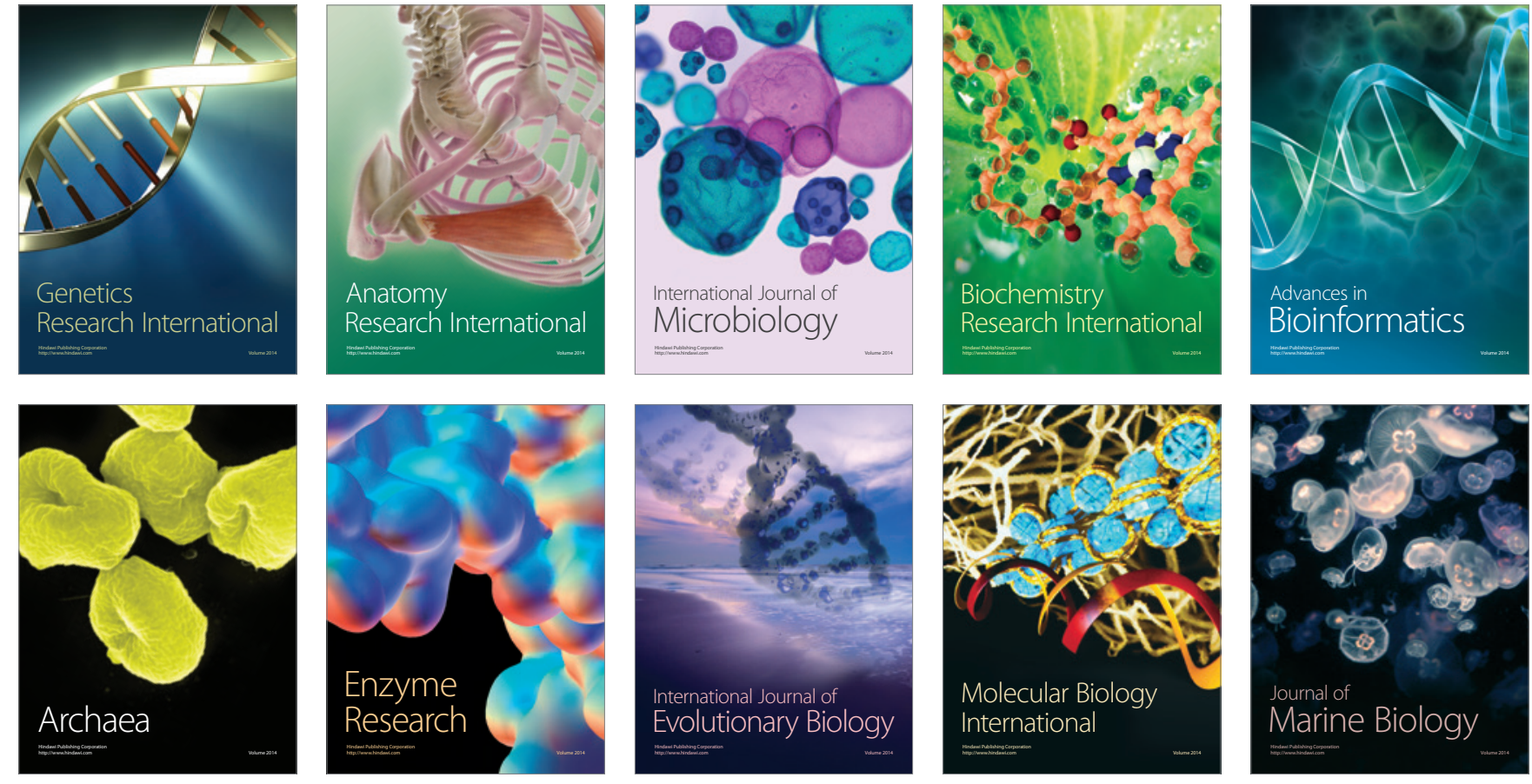